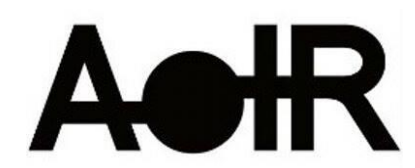

Selected Papers of \#AolR2021:

The 22nd Annual Conference of the

Association of Internet Researchers

Virtual Event / 13-16 Oct 2021

\title{
VERTICAL OR HORIZONTAL? AN EMPIRICAL INVESTIGATION OF THE ROLE OF PRIVACY LITERACY AND PRIVACY SELF-EFFICACY ON THE DIMENSIONALITY OF PRIVACY
}

\author{
Dmitry Epstein \\ Hebrew University of Jerusalem \\ Kelly Quinn \\ University of Illinois at Chicago
}

Privacy is increasingly acknowledged as a multidimensional and expansive phenomenon. Empirical evidence of inconsistencies between expressed levels of concerns for privacy and engagement in privacy-protecting behavior (PPB) - the socalled "privacy paradox - motivated scholars to adopt a more nuanced approach towards this relationship. Recent research has differentiated between peer-facing (horizontal) privacy relationships from those oriented towards institutions and service providers (vertical privacy) (Quinn et al., 2019; Raynes-Goldie, 2010). Such dimensionality is frequently overlooked when examining PPB, leading to orthogonal understandings of how individuals navigate privacy on a daily basis. The fundamental relationship between privacy concerns and PPB is further complicated by mediating and moderating factors, such as digital privacy literacy and privacy self-efficacy, or the belief in one's ability to achieve a desired level of privacy (Chen \& Chen, 2015; Dienlin \& Metzger, 2016; Masur, 2020). In a social media context, where users trade personal information privacy with platform providers in exchange for services that support peer communication and relational maintenance, research, has typically counterposed vertically-oriented privacy concerns against horizontally-oriented disclosure behaviors (Quinn et al., 2019). Rarely have these two privacy perspectives been examined consistently or in tandem.

The goals of this study are two-fold. We extend established models linking attitudes related to privacy concerns and PPB by (a) differentiating between horizontal (social) and vertical (institutional) orientations of PPB as capturing an aspect of privacy multidimensionality, and (b) introducing additional explanatory factors such as privacy literacy and privacy self-efficacy into the modeling of PPB.

\section{Method}

Suggested Citation (APA): Epstein, D. \& Quinn, K. (2021, October). Vertical or horizontal? An empirical investigation of the role of privacy literacy and privacy self-efficacy on the dimensionality of privacy. Paper (or panel) presented at AolR 2021: The 22nd Annual Conference of the Association of Internet Researchers. Virtual Event: AolR. Retrieved from http://spir.aoir.org. 
We test these relationships by focusing specifically on social media as a communicative environment, as opposed to general privacy online. Empirically, this work builds on a representative sample ( $n=686$ ) of social media users in the US, matched to the 2015 US Census Bureau's American Community Survey on the parameters of income, gender, and age. We use confirmatory factor analysis to validate the measurement models for the latent constructs of privacy concern (Xu et al., 2011), trust (Krasnova et al., 2012), privacy self-efficacy (Dienlin \& Metzger, 2016), and vertical and horizontal PPB. As a measured variable, we introduce the construct of privacy literacy (Masur, 2020), which captures four domains of privacy knowledge--institutional practices, technical aspects of privacy protection, privacy law, and privacy protection strategies--for its influence on privacy concerns and trust. To model the resulting relationships, we employed covariance-based structural equation modeling.

\section{Findings}

Analysis of the data demonstrates that PPB can be dimensionalized as vertical and horizontal aspects of privacy (Figure 1). Although these two orientations are strongly correlated, privacy concerns have differing relationships with each. Traditional privacy concerns have a stronger positive relationship with horizontal PPB than with vertical PPB. Trust in the platform, which is inversely/negatively correlated with privacy concern, is also positively related to PPB, though it is somewhat equivalent in its effects on both vertical and horizontal behaviors. Suggesting that PPB is enhanced by higher levels of trust in the platform operator is initially surprising, but it may also mean that the relationship between trust and PPB is mediated through additional factors, such as privacy self-efficacy. 
Figure 1 - Dimensionalized PPB*

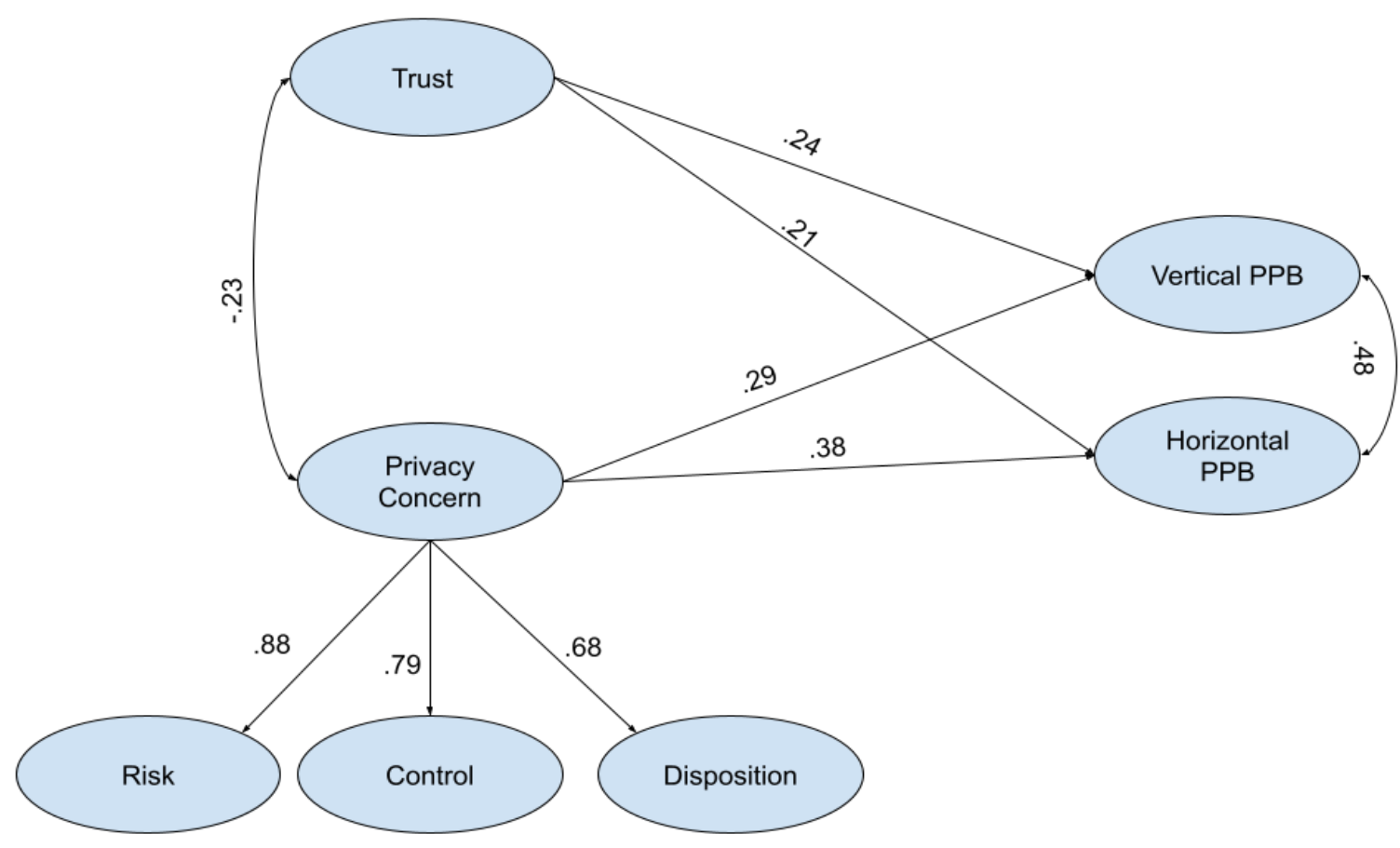

*Standardized estimates are displayed

The addition of privacy literacy and privacy self-efficacy suggests they are important factors in explaining both vertical and horizontal PPB (Figure 2). First, online privacy literacy has both a substantive positive effect on privacy concerns and a substantive negative effect on an individual's trust in the social media platform. In other words, as privacy literacy increases, both privacy concerns are triggered and trust in the platform provider declines. This is important, as both privacy concern and trust in platform providers are critical factors in explaining PPB. 

privacy*

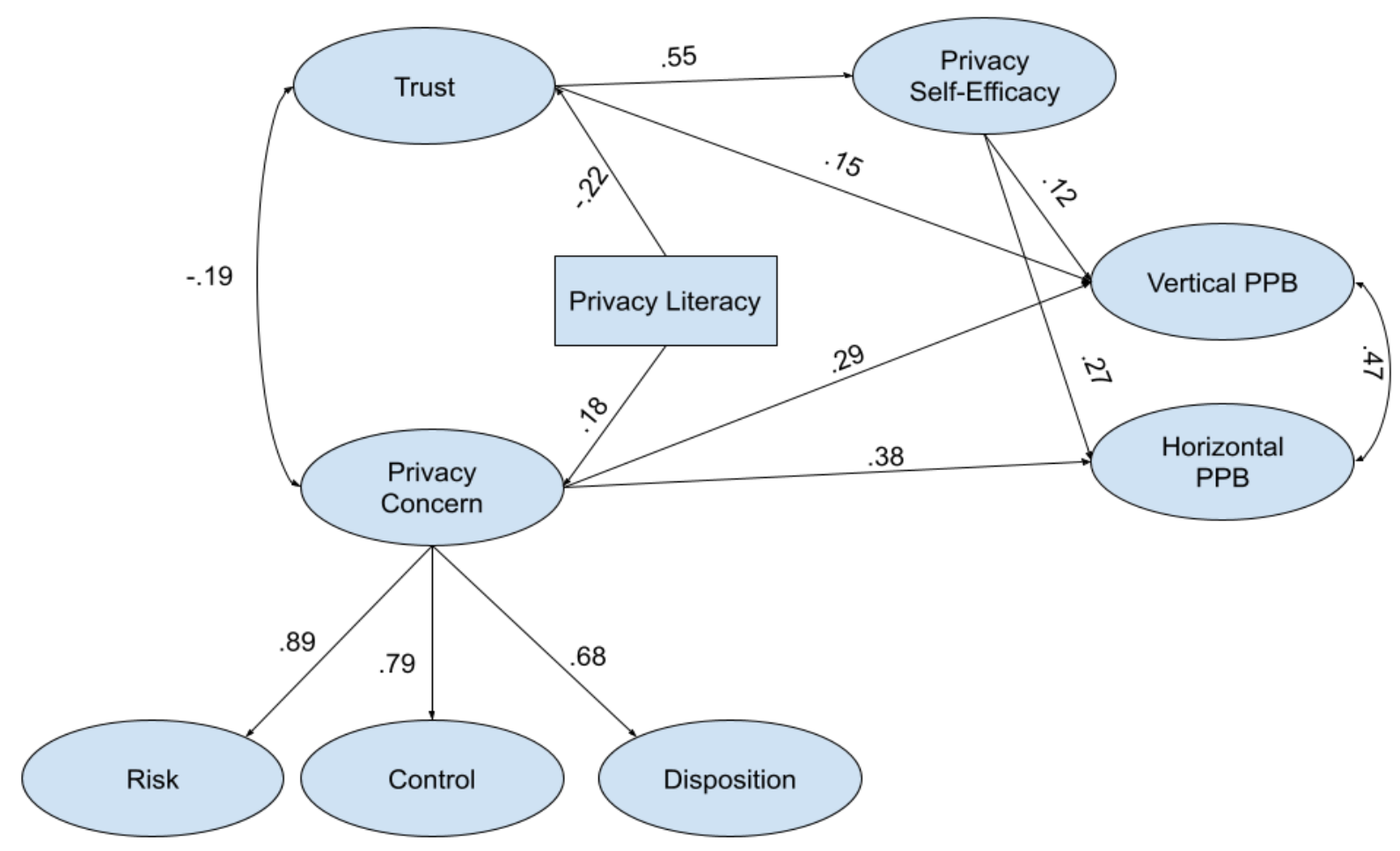

${ }^{*}$ Standardized estimates are displayed

Second, privacy self-efficacy emerges as an important factor, as its strong association with trust helps to partially explain the initially surprising relationship between trust and PPB. Introduction of privacy self-efficacy to the model eliminates the direct role that trust seemed to play in explaining horizontal PPB and it partially mediates the role of trust in explaining vertical PPB. On the one hand, the differential role of privacy self-efficacy in explaining vertical and horizontal PPB further supports the hypothesis that PPB can be dimensionalized. On the other hand, the mediated relationship between trust and horizontal PPB suggests that trust in a platform provider in fact does not predict the actual PPB, but rather one's belief in their ability to protect social privacy on the platform. This observation seems in line with the rhetoric of platform providers, who emphasize the controls they offer their users for management of horizontal relationships.

At the same time the more complex constellation of direct and mediated relationships between trust, privacy self-efficacy and vertical PPB, remains somewhat puzzling. The fact that privacy self-efficacy has a much weaker positive relationship with vertical, compared with horizontal, PPB is not surprising given our observation above. Yet, the direct positive, albeit weak, relationship between trust and vertical PPB is not intuitive, as we would not expect it to be significant. It is possible that the factors that mediate the link between trust in platform providers and horizontal PPB differ from those that 
mediate that link with vertical PPB as the two are based on different threat models. Alternatively, it is possible that we need a more nuanced measure of trust - one that accounts for the dimensionality of the phenomenon similarly to our treatment of privacy in this study.

\section{Implications}

This work reaffirms the basic logic of the relationship between privacy concerns and PPB, and expands its explanatory power by incorporating literacy and self-efficacy as relevant factors. We argue that a move from unidimensional views of privacy will encourage new ideas in the realms of privacy technology design, regulation, and activism. Both our findings about the relationship between privacy concerns and the orientation of PPB and the roles that privacy self-efficacy and privacy literacy play in PPB, highlight that vertical and horizontal dimensions of privacy are enacted through different mechanisms. This is particularly important as we recognize that users of social media tend to adopt horizontal views of privacy (Quinn et al., 2019), whereas many systemic threats stem from vertical privacy relationships (Baruh \& Popescu, 2017). In this regard, the negative relationships between privacy literacy and privacy self-efficacy with vertical PPB warrants particular attention and further exploration to understand the mechanisms underlying those relationships.

\section{References}

Baruh, L., \& Popescu, M. (2017). Big data analytics and the limits of privacy selfmanagement. New Media \& Society, 19(4), 579-596. https://doi.org/10.1177/1461444815614001

Chen, H.-T., \& Chen, W. (2015). Couldn't or wouldn't? The influence of privacy concerns and self-efficacy in privacy management on privacy protection. Cyberpsychology, Behavior, and Social Networking, 18(1), 13-19. https://doi.org/10.1089/cyber.2014.0456

Dienlin, T., \& Metzger, M. J. (2016). An extended privacy calculus model for SNSsAnalyzing self-disclosure and self-withdrawal in a U.S. representative sample. Journal of Computer-Mediated Communication, 21(5), 368-383. https://doi.org/10.1111/jcc4.12163

Krasnova, H., Veltri, N. F., \& Günther, O. (2012). Self-disclosure and privacy calculus on social networking sites: The role of culture. Business \& Information Systems Engineering, 4(3), 127-135.

Masur, P. K. (2020). How online privacy literacy supports self-data protection and selfdetermination in the age of information. Media and Communication, 8(2), 258269. https://doi.org/10.17645/mac.v8i2.2855

Quinn, K., Epstein, D., \& Moon, B. (2019). We care about different things: Non-elite conceptualizations of social media privacy. Social Media + Society, 5(3). https://doi.org/10.1177/2056305119866008

Raynes-Goldie, K. (2010). Aliases, creeping, and wall cleaning: Understanding privacy in the age of Facebook. First Monday, 15(1). http://firstmonday.org/ojs/index.php/fm/article/view/2775

Xu, H., Luo, X., Carroll, J. M., \& Rosson, M. B. (2011). The personalization privacy paradox: An exploratory study of decision making process for location-aware marketing. Decision Support Systems, 51(1), 42-52. https://doi.org/10.1016/j.dss.2010.11.017 